\title{
SPINAL CORD INVOLVEMENT IN BRACHIAL PLEXUS INJURY
}

The role of spinal cord plasticity after birth injury and recovery from obstetric brachial plexus lesions was investigated in newborn rats with selective crush injury to spinal roots $\mathrm{C} 5$ and $\mathrm{C} 6$, in a study at University Clinics of Vienna School of Medicine, Austria. Evaluation of recovery of function following motoneuron loss, using the Bertelli test of grooming, grid walk, retrograde tracing of motoneuron pools, and functional muscle testing, showed that the adjacent C7 motoneuron contribution to biceps muscle innervation increased 4-fold after upper trunk lesions, compensating for the motoneuron loss from injury. (Korak KJ, Tam SL, Gordon T, et al. Changes in spinal cord architecture after brachial plexus injury in the newborn. Brain 2004;127:1488-1495). (Respond: Oskar C Aszmann MD, Division of Plastic and Reconstructive Surgery, Department of Surgery, University Clinics of Vienna School of Medicine, Waehringer Guertel 18-20, 1090 Vienna, Austria).

COMMENT. In newborns with obstetric brachial plexus palsy affecting spinal roots C5 and C6, an intact C7 innervation of the biceps muscle is essential for a recovery process to ensue. The loss of motoneurons in C5 and C6 spinal cord segments is compensated for by changes in the spinal cord architecture and an increased contribution of $\mathrm{C} 7$ motoneurons.

\section{SEIZURE DISORDERS}

\section{PARTIAL EPILEPSY WITH AUDITORY FEATURES}

The clinical characteristics of 53 sporadic (S) cases of idiopathic partial epilepsy with auditory features (IPEAF) were analyzed and compared to previously reported familial (F) cases of autosomal dominant partial epilepsy with auditory features (ADPEAF) in a study at the University of Bologna, Italy. Age at onset of seizures ranged from 6 to 39 years (aver. 19 years), and the most common type were secondarily generalized (79\%). Auditory auras occurred alone $(53 \%)$ or were associated with visual, psychic or aphasic symptoms. Most auditory auras were simple hallucinations of sound, like hissing, buzzing and vibration. Forty percent described complex, well-formed acoustic symptoms, like music or human voices. Loss of hearing was reported in $25 \%$. Symptoms could be lateralized to one ear in $28 \%$ and were bilateral in $15 \%$.. Sudden noise, a flushing toilet or answering the phone triggered the aura in 5 patients. The EEG was epileptiform in 18 (34\%), and showed temporal slowing in $27(51 \%)$. NMR scans in 47 and CT in 6 were normal except for minor asymmetries of ventricles in 6 . Seizures were controlled in $51 \%$, but tended to recur after drug withdrawal. The clinical characteristics of S and F patients were similar in age at onset, seizure frequency and response to therapy. Unlike cases of ADPEAF, mutations in LG11/epitempin exons were absent and the family history was negative in IPEAF. IPEAF is a form of temporal lobe epilepsy closely related to ADPEAF, and the majority of cases have a benign course. (Bisulli F, Tinuper P, Avoni P, et al. Idiopathic partial epilepsy with auditory features (IPEAF): a clinical and genetic study of 53 sporadic cases. Brain 2004;127:1343-1352). (Respond: Paolo Tinuper MD, Department of Neurological Sciences, University of Bologna, Via Ugo Foscolo 7, 40123 Bologna, Italy). 
COMMENT. IPEAF is a benign form of temporal lobe epilepsy without structural brain lesion or known cause. The authors attach the term 'idiopathic' because they consider some unrecognized genetic factor might play a role in etiology. The authors are to be commended on their historical review of auditory seizures that includes the early references of Holmes (1927), Lennox (1960), Penfield (1951), and Currie (1971). Interest in auditory partial epilepsy has been renewed following the report of a syndrome of autosomal dominant partial epilepsy with auditory features (Ottman et al, 1995).

\section{EPILEPSY OUTCOME IN PERIVENTRICULAR NODULAR HETEROTOPIA}

The clinical, electroencephalographic, and neuroimaging features and course of seizures in 16 patients with periventricular (subependymal) nodular heterotopia (PNH) were investigated at the University of Bologna, Italy. The mean follow-up was 17.3 years, mean age at follow-up 29 years (range 15-48 years). Simple cases of PNH ( 8 cases; 5 women and 3 men) were characterized by normal intelligence and seizures that were usually partial, associated with focal EEG, began in the second decade (mean age at seizure onset 19.2 years (range 17-22), were infrequent and tended to disappear. The 8 complicated PNH plus cases ( 3 women and 5 men) were distinguished by mental retardation and seizures that began in the first decade (mean 4.25 years; range 1-14), associated with focal and bisynchronous EEG abnormalities, were very frequent and multiple in pattern (partial, secondarily generalized, akinetic or tonic clonic), and often medically refractory. The MRI in simple PNH cases showed only periventricular nodules, whereas PNH plus cases were characterized by other brain malformations, including subcortical heterotopia, polymicrogyria, focal dysplasia, schizencephaly, cortical infolding, agenesis of the corpus callosum, mega cisterna magna and cerebellar atrophy. (d'Orsi G, Tinuper P, Bisulli F, et al. Clinical features and long term outcome of epilepsy in periventricular nodular heterotopia. Simple compared with plus forms. J Neurol Neurosurg Psychiatry 2004;75:873-878). (Respond: Professor P Tinuper, Department of Neurological Sciences, University of Bologna, Via Ugo Foscolo 7, 40123 Bologna, Italy).

COMMENT. Patients with seizures associated with periventricular nodular heterotopia (PNH) are classified in two groups: 1) Simple PNH and 2) PNH plus. The PNH plus cases characterized by additional brain malformations have an earlier presentation and carry a poor prognosis.

Genetics of periventricular heterotopia. Four families of $\mathrm{PNH}$ are reported in which FLN1 mutations caused PNH in men. In 2 families mild missense mutations were transmitted from mother to son and from a father to daughter, causing mild phenotypes, with unilateral PNH in one. In a third family, an affected man with classic PNH (bilateral PNH, enlarged cisterna magna, and cerebellar vermis hypoplasia) had mosaic mutations of FLN1 gene, and in the fourth family, a truncating mutation was associated with early postnatal death of a boy with $\mathrm{PNH}$, focal polymicrogyria, and cardiovascular and genitourinary malformations. X-linked PNH caused by FLN1 mutations in men is clinically heterogeneous and caused by different genetic mechanisms. (Guerrini R, et al. Neurology July (1 of 2) 2004;63:51-56). Affected women usually have epilepsy and normal or borderline intelligence. 